Article

\title{
Broadband Asymmetric Propagation in Pillared Meta-Plates
}

\author{
Lin Chen ${ }^{1}$, Wei Wang ${ }^{2, *}$, Guohua Nie ${ }^{1}$, Yabin Jin ${ }^{1, *}$, Daniel Torrent ${ }^{3, *}$ and \\ Bahram Djafari-Rouhani ${ }^{4, *}$ (1)
}

1 School of Aerospace Engineering and Applied Mechanics, Tongji University, Shanghai 200092, China; linlinchen@tongji.edu.cn (L.C.); ghnie@tongji.edu.cn (G.N.)

2 Institut des NanoSciences de Paris, Sorbonne Université, UPMC Université Paris 06 (INSP-UMR CNRS 7588), 4 Place Jussieu, 75005 Paris, France

3 GROC-UJI, Institut de Noves Tecnologies de la Imatge, Universitat Jaume I, 12080 Castello, Spain

4 Département de Physique, Institut d'Electronique, de Microélectonique et de Nanotechnologie, Université de Lille, UMR CNRS 8520, 59650 Villeneuve d'Ascq, France

* Correspondence: wwangscorpio@hotmail.com (W.W.); 083623jinyabin@tongji.edu.cn (Y.J.); dtorrent@uji.es (D.T.); bahram.djafari-rouhani@univ-lille.fr (B.D.-R.)

Received: 9 July 2020; Accepted: 12 August 2020; Published: 14 August 2020

check for updates

\begin{abstract}
The asymmetric propagation of mechanical energy across interfaces is a challenging problem with a wide range of applications. In this work, we present a novel structure presenting the asymmetric propagation of elastic waves in thin plates in a broadband range. The structure consists of a combination of symmetrically and asymmetrically distributed pillars, so that the former decouple the different Lamb modes and the latter mix all of them. We show that a combination in tandem with these two structures can realize an efficient broadband asymmetric propagation at the subwavelength range and achieve a transmission difference larger than $200 \mathrm{~dB}$ between forward and backward directions. The proposed pillared meta-plate brings a new way for subwavelength and broadband wave manipulation in the fields of wave isolation, sensing and communication, among others.
\end{abstract}

Keywords: phononic crystals; Lamb wave; asymmetric transmission

\section{Introduction}

In the past decade, pillared phononic crystal plates [1,2], consisting of a periodic arrangement of pillars deposited on a thin plate, have attracted increasing attention since they can exhibit band gaps originating from both Bragg scattering, when the wavelength is comparable to the lattice constant, and from the local resonances of the pillars at wavelengths which are much larger than the lattice constant. Therefore, these structures can be regarded as phononic crystals [3,4] or an acoustic metamaterial [5-7] according to the operating wavelengths. For the latter case, if the thickness of the plate and that of the pillars is smaller than the operating wavelength, they can be defined as an acoustic metasurface [8-11], which is a compact structure that is able to steer waves with advanced functions. The multiple roles of the pillared plate gives rise to fundamental scientific interests and diverse applications such as waveguiding [12-14], sensing [15-17], lensing [7,18], and topological insulations [19-25], among others.

Unidirectional structures that allow acoustic or elastic energy passing only in one direction mimicking the behavior of diodes have been receiving significant attention recently. For conventional materials, acoustic waves can be transmitted symmetrically from both sides due to the reciprocity principle based on time-reversal symmetry. Many efforts have dealt with the design of non-reciprocal devices based on different proposed mechanisms [26]. A ring resonator with air circulation-flow has 
been proposed to show nonreciprocal sound transmission among three ports [27]. Instead of fluid motion, spatiotemporal modulation was introduced in phononic crystals or metamaterials to achieve a nonreciprocal acoustic transmission [28-33]. Nonlinearity opens another route with frequency conversion in a nonlinear material associated with a band gap material as a filter [34-37]. Another route is based on the unidirectional transmission in lossy acoustic metasurfaces originating from the loss-induced suppression of acoustic beams in high order diffractions [38]. Mode conversions and filtering were also proposed in a linear system to exhibit a unidirectional transmission [39]. With acoustic parity-time-symmetric metamaterials, a unidirectional transmission was also demonstrated at the exceptional point [40]. Recently, a high transmission efficiency for an air-borne sound wave unidirectional transmission has been realized by combining mode transition, filtering and cavity resonance [41].

Some general limitations in the above asymmetric propagation devices can be detrimental for their potential applications, especially for elastic waves. For instance, they can have a narrow operating bandwidth, bulky structures, low transmission efficiency, or complex geometries. Therefore, from a practical point of view, subwavelength and broadband devices with high transmission efficiencies and simple geometries are strongly desired in asymmetric propagations for elastic wave isolations, nondestructive evaluations and signal processing.

In this work, we investigate subwavelength and broadband asymmetric elastic propagation in a pillared meta-plate. It consists of an association in tandem with two pillared plate arrays where the pillars are distributed respectively, symmetrically and asymmetrically with respect to the mid-plane of the thin plate. Without a loss of generality, we assume a square lattice arrangement of the pillars in each array. In the symmetric-sided pillars, the symmetric and antisymmetric Lamb modes are decoupled from each other, while in the asymmetric-sided pillars they are mixed together.

The paper is organized as follows: After this introduction, in Section 2, the mechanism for asymmetric propagation based on mode-hybridization and broadband low-frequency gaps is explained, where either for $\mathrm{S}_{0}$ or $\mathrm{A}_{0}$ Lamb modes this structure will allow the transmission of these modes in the forward direction while being prohibited from going in the backward direction. It will be shown that these phenomena can occur at the subwavelength range and, moreover, that the entire length of the pillared meta-plate can be designed to remain subwavelengths, making it a compact metasurface for wave isolation and signal processing at the micro scale. In Section 3 we present the dispersion curves and transmission spectra of each array of the pillared plate, and in Section 4 we discuss the phenomenon of asymmetric propagation when the two pillared arrays are associated in tandem. In Section 5, some alternative designs displaying the asymmetric propagation are discussed and a summary of the work is presented in Section 6.

\section{Mechanism of Asymmetric Transmission in Pillared Meta-Plates}

We considered a meta-plate consisting of periodic pillars in a square lattice deposited on a thin plate, either symmetrically or asymmetrically, as shown in Figure 1. The lattice constant was $a=$ $100 \mu \mathrm{m}$ and the plate's thickness was $e=80 \mu \mathrm{m}$. The pillars in both symmetric and asymmetric parts were identical with a height $h_{1}=h_{2}=320 \mu \mathrm{m}$ and a diameter $d_{1}=d_{2}=80 \mu \mathrm{m}$. The entire geometry was made of isotropic steel, with the elastic modulus $E=200 \mathrm{GPa}$, Poisson's ratio $v=0.3$ and mass density $\rho=7850 \mathrm{~kg} / \mathrm{m}^{3}$. Perfectly matched layers (PML) were placed at the two ends to reduce the wave reflection from boundaries. The transmission calculations throughout this work were implemented with the frequency domain.

The propagation of waves in a symmetric plate is described in terms of symmetric (S) and antisymmetric (A) Lamb modes plus the shear polarization (SH). Assuming propagation along the $x$ direction, and that the plate is contained in the $x-y$ plane, the $\mathrm{S}$ and A modes present a dominant $x$ and $z$ component, respectively [18]. In the symmetric pillared plate, the modes can also be decoupled between symmetric and anti-symmetric modes with respect to the symmetry plane in the middle plane of the plate. In contrast, this symmetry does not hold for the one-sided pillared structure and there is 
no decoupling between its modes based on symmetry argument. For this reason, for the structure depicted in Figure 1, where these two structures are merged, if an $\mathrm{S}_{0}\left(\right.$ resp. $\mathrm{A}_{0}$ ) mode is launched in the forward direction, it can excite all the modes of the one-sided pillared structure, while the same wave launched in the backward direction is able to excite only the modes of the pillared structure, since these have the same symmetry. Now, assume that the frequency of the incident wave belongs to a symmetric (anti-symmetric) wave bandgap of the two-sided pillared structure. Therefore, it cannot go in the backward direction. However, in the forward direction it excites all the modes of the one-sided pillared structure and leaves this part of the system with both symmetric and anti-symmetric components. Consequently, the anti-symmetric (symmetric) component can traverse the two-sided pillared structure. In this way, asymmetric propagation is achieved for either an $\mathrm{S}_{0}$ or $\mathrm{A}_{0}$ incident wave.

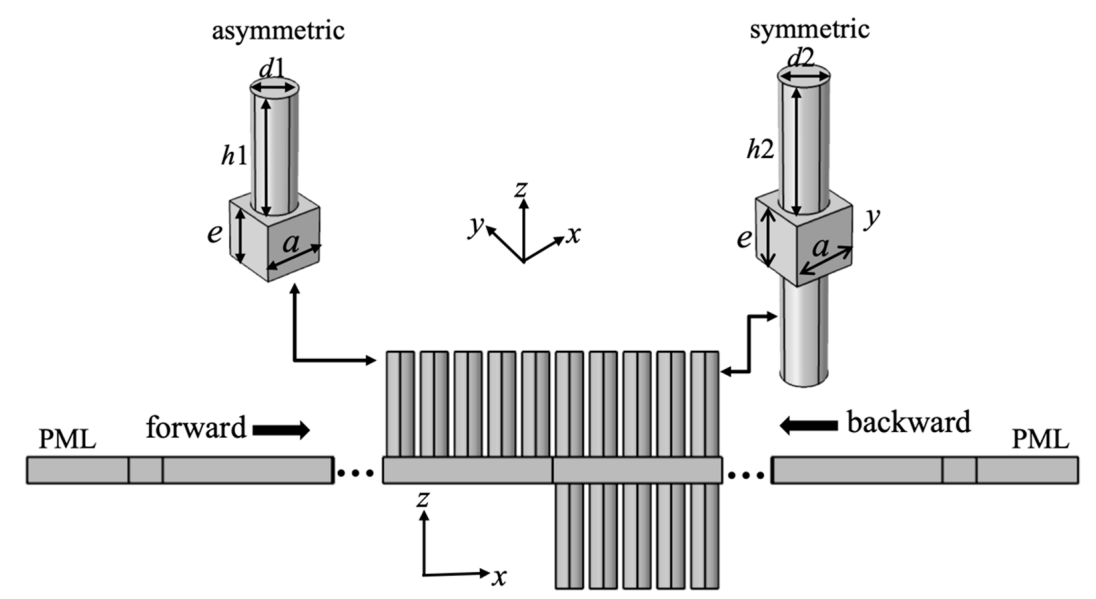

Figure 1. Schematic of the pillared meta-plate consisting of symmetric- and asymmetric-sided pillars on a thin plate arranged in a square array of lattice constant $a$. Each pillar has a diameter $d$ and a height $h$. The plate has a thickness $e$. The forward and backward propagating directions are defined along the $+x$ and $-x$ axis, respectively.

It is worth noting that the choice of the geometrical parameters achieves a broadband and subwavelength device with a total length of the device smaller than the typical operating wavelength. Indeed, for a frequency of $3 \mathrm{MHz}$, the wavelength of the $S_{0}$ Lamb mode wave is about $2000 \mu \mathrm{m}$, which is 20 times bigger than the lattice constant. Additionally, the whole size of the device can be kept below the wavelength. In general, a periodic structure with a minimum length of $5 \sim 6$ lattice constants is sufficient to exhibit bandgaps and hybridized bands, so that the entire length of asymmetric and symmetric structures along $x$ can be constituted by less than 15 unit cells, which is still smaller than one wavelength in the background plate. Recent work on asymmetric transmission with acoustic metasurfaces [42,43] remarks on the great advantage of subwavelength devices such as the ones we propose here. Finally, the design proposed here can be efficient with a broad bandwidth because the gaps of the symmetric pillared structure can be made sufficiently broad.

\section{Dispersion Curves and Transmission of Symmetric and Asymmetric Pillared Meta-Plates}

The dispersion curve of the infinite periodic plate provides a good reference for the transmission properties of the finite stacked structures. The unit cell of each structure is shown in the zoom-in upper panel of Figure 1, with periodic boundary conditions for the interfaces between the neighboring unit cells based on the Bloch theorem as

$$
\boldsymbol{u}(\boldsymbol{r}+a)=\boldsymbol{u}(\boldsymbol{r}) e^{i(\boldsymbol{k} a)}
$$

where $u$ is the displacement vector, $\boldsymbol{r}$ is the position vector, and $\boldsymbol{k}$ is the Bloch wave vector. In general, eigenvalue equations of this periodic structure can be described in the following form

$$
\left(\boldsymbol{K}-\omega(k)^{2} \boldsymbol{M}\right) \boldsymbol{u}=0
$$


where the matrix $K$ and $M$ refer to stiffness and mass, respectively. For square lattices, the wavevector should be swept along -X-M- within the first irreducible Brillouin zone, so that the eigenvalue equations will be solved by the finite element method to obtain the dispersion curves $\omega=\omega(\boldsymbol{k})$ of the structure.

We firstly calculate the dispersion and transmission properties of the symmetric pillared meta-plate (Figure 2). It is widely demonstrated that definitions such as $\left|u_{z}\right|\left|u_{z r e f}\right|$ and $\left|u_{x}\right|\left|u_{x r e f}\right|$ can efficiently characterize the transmission coefficient for $\mathrm{A}_{0}$ and $\mathrm{S}_{0}$ Lamb waves, respectively, where the $\left|u_{x}\right|\left(\left|u_{z}\right|\right)$ are detected on the pillared plate in the downstream zone and $\left|u_{x r e f}\right|\left(\left|u_{z r e f}\right|\right)$ are detected on the reference plate without any pillar at the same position, given that the dominant displacement component for the $\mathrm{S}_{0}$ and $\mathrm{A}_{0}$ modes are $u_{x}$ and $u_{z}$, respectively. From the comparison between the dispersion and the transmission curves in Figure 2, the polarization bandgaps correspond well to the frequency ranges with almost zero transmission, proving the correctness of the transmission definition. The adopted model is shown in the inset of the right panel. PMLs are employed at two ends along the $x$ direction to eliminate the reflections from the boundaries. In between, there are 15 symmetric-sided unit cells. Moreover, periodic conditions are applied on the lateral faces along the $y$ direction to simulate the wave propagation along the $\Gamma X$ direction. As explained above, the dispersion curves (left panel of Figure 2) can be decoupled into symmetric (orange) and antisymmetric (blue) modes with respect to the middle plane of the plate thanks to the geometric symmetry, which clearly exhibits the bandgaps for these two kinds of modes. As an example, we present in the insets of Figure 2 the field of the antisymmetric eigenmode shown by a green dot on the dispersion curves. Here, one can notice the antisymmetric behavior of both real parts of $u_{x}$ and $u_{z}$. In the transmission calculation (right panel of Figure 2), we consider both $\mathrm{S}_{0}$ (orange) and $\mathrm{A}_{0}$ (blue) Lamb wave excitations whose results are in good agreement with the dispersions. In particular, the wave propagation is prohibited for the symmetric modes in Frequency Zones 1 and 3, occurring at the intervals 2.9-3.5 and 7-7.7 MHz, respectively, while the antisymmetric modes are allowed to propagate. The opposite situation occurs in Frequency Zone 2 at 3.8-6 MHz.
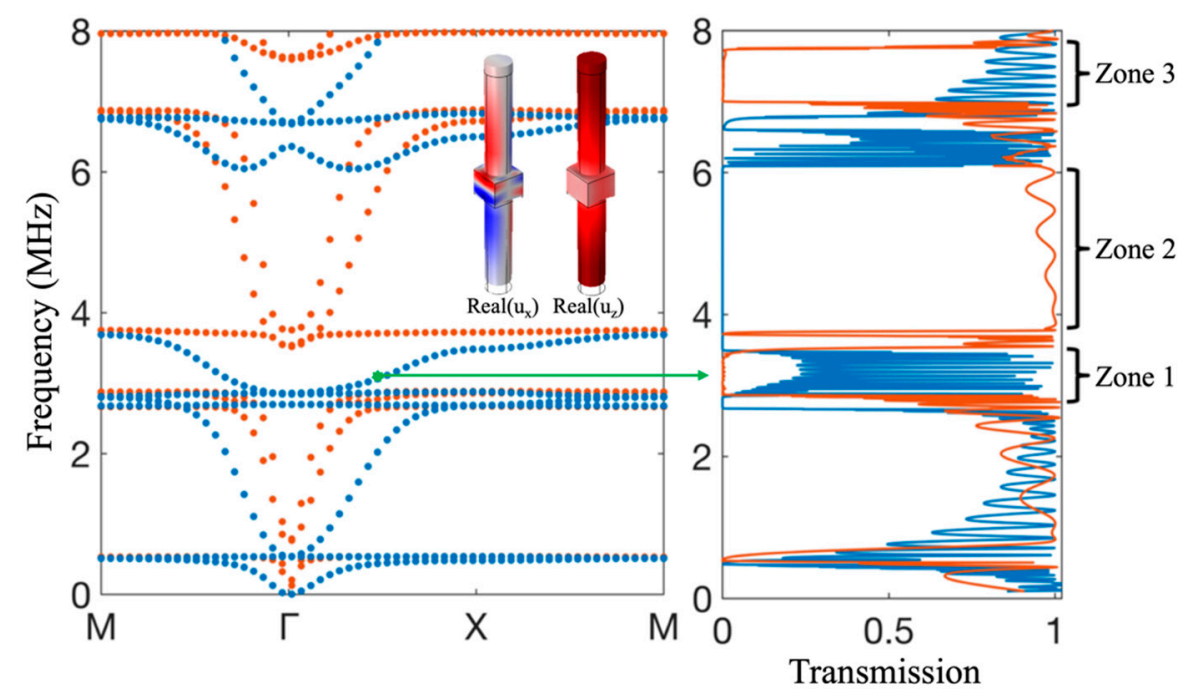

Figure 2. Left panel: Dispersion curves of the symmetric-sided pillared meta-plate along the first irreducible Brillouin zone. The orange and blue bands represent the symmetric and antisymmetric Lamb modes with respect to the middle plane of the plate, respectively. Right panel: Transmission spectra of $\mathrm{A}_{0}$ (blue) and $\mathrm{S}_{0}$ (orange) Lamb waves across 15 symmetric-sided unit cells along the $\Gamma X$ direction (see the inset). The wave propagation in not allowed for the $S_{0}$ Lamb wave in Frequency Zone $1(2.9-3.5 \mathrm{MHz})$ and Zone $3(7-7.7 \mathrm{MHz})$, and for $\mathrm{A}_{0}$ Lamb wave in Frequency Zone 2 (3.8-6 MHz), which match well with the bandgaps of corresponding symmetries. The real parts of $u_{x}$ and $u_{z}$ of the antisymmetric eigenstate at the green dot in the dispersion curves are plotted as the insets, where the red and blue colors mean positive and negative values, respectively. 
For the asymmetric pillared meta-plate, the dispersion curves shown in Figure 3 have similar bands as in Figure 2. However, symmetric and antisymmetric modes can no longer be distinguished. Both of the cross-section fields of real $\left(u_{z}\right)$ and real $\left(u_{x}\right)$ are in phase for the eigenmode at the green dot in the dispersion, so that it allows the propagation for both modes as pointed to by the arrow. This can be noticed in the transmission curves shown in the right panel of Figure 3, using the same definition as in Figure 2. Indeed, Figure 3 shows that both the $S_{0}$ and $A_{0}$ Lamb waves can be transmitted in Frequency Zones 1-3, which were forbidden bands for one symmetry in Figure 2 . This is due to the fact that an incident $S_{0}$ or $A_{0}$ wave will generate in the phononic crystal modes which do not obey the symmetry rule, hence allowing the simultaneous transmission of the $\mathrm{S}_{0}$ and $\mathrm{A}_{0}$ waves after leaving the phononic crystal. Therefore, the geometry shown in Figure 1, where this asymmetric pillared structure is associated in tandem with symmetric one functioning as the metamaterial filter, is able to realize asymmetric propagation, in the sense that the same polarization cannot carry out the same energy when traveling from the opposite sides of the structure.
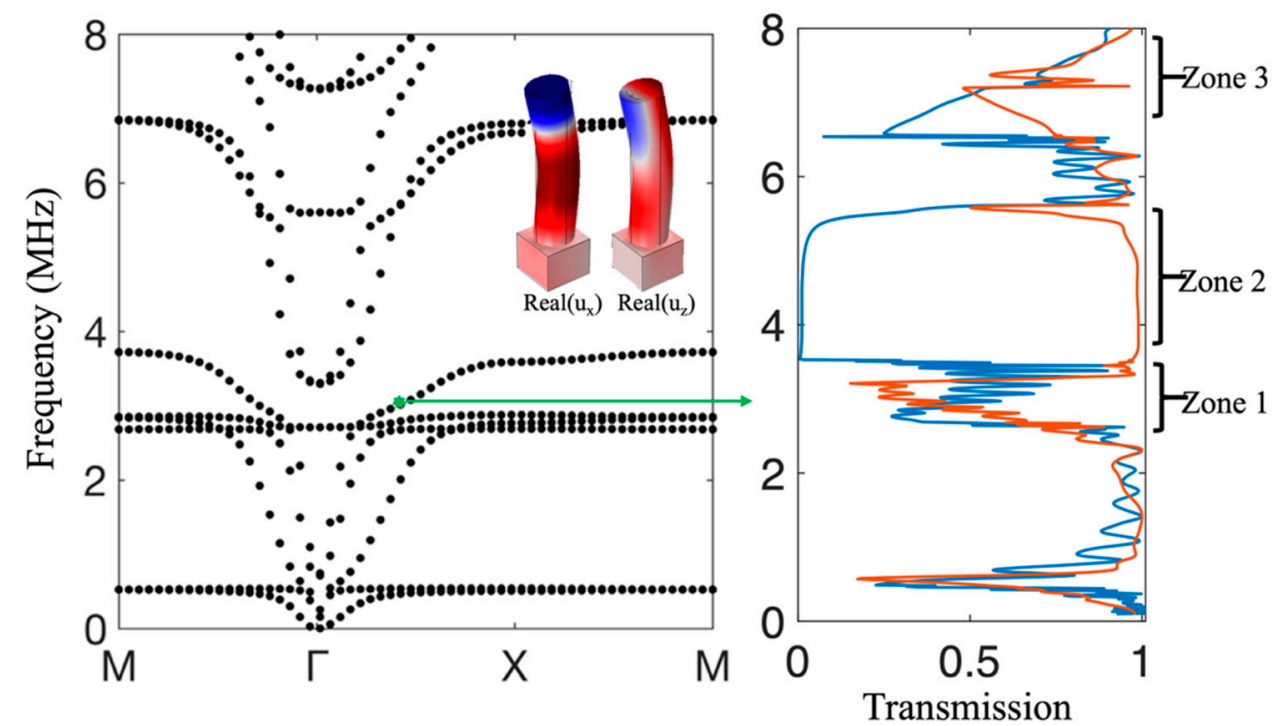

Figure 3. Left panel: Dispersion curves of the asymmetric-sided pillared meta-plate along the first irreducible Brillouin zone. Due to the asymmetry with respect to the middle plane of the plate, the dispersion curves cannot be decoupled, and behave as hybridized bands. Right panel: Transmission spectra of $\mathrm{A}_{0}$ (blue) and $\mathrm{S}_{0}$ (orange) Lamb waves across 15 asymmetric sided unit cells along the $\Gamma X$ direction. The transmissions in Frequency Zones 1-3 change from those in Figure 2, with non-vanishing values for both Lamb modes. The real parts of $u_{x}$ and $u_{z}$ of the eigenstate at the green dot in the dispersion curves are plotted as the insets where the red and blue colors mean positive and negative values, respectively.

To give a clear picture about how the mode conversion occurs after transmission through the asymmetric-pillared meta-plate, we present the discrete fast Fourier transform (FFT) of the out-of-plane displacements $u_{z}$ on top of the plate at $5 \mathrm{MHz}$ in Figure 4. The left and right panels refer to an $\mathrm{A}_{0}$ incident wave and to the transmitted waves, respectively. While the incident wave is characterized by a single $\mathrm{A}_{0}$ peak, one can notice two peaks including both the $\mathrm{A}_{0}$ and $\mathrm{S}_{0}$ modes in the transmitted wave, resulting from the partial conversion of the incident wave into an $S_{0}$ wave. 


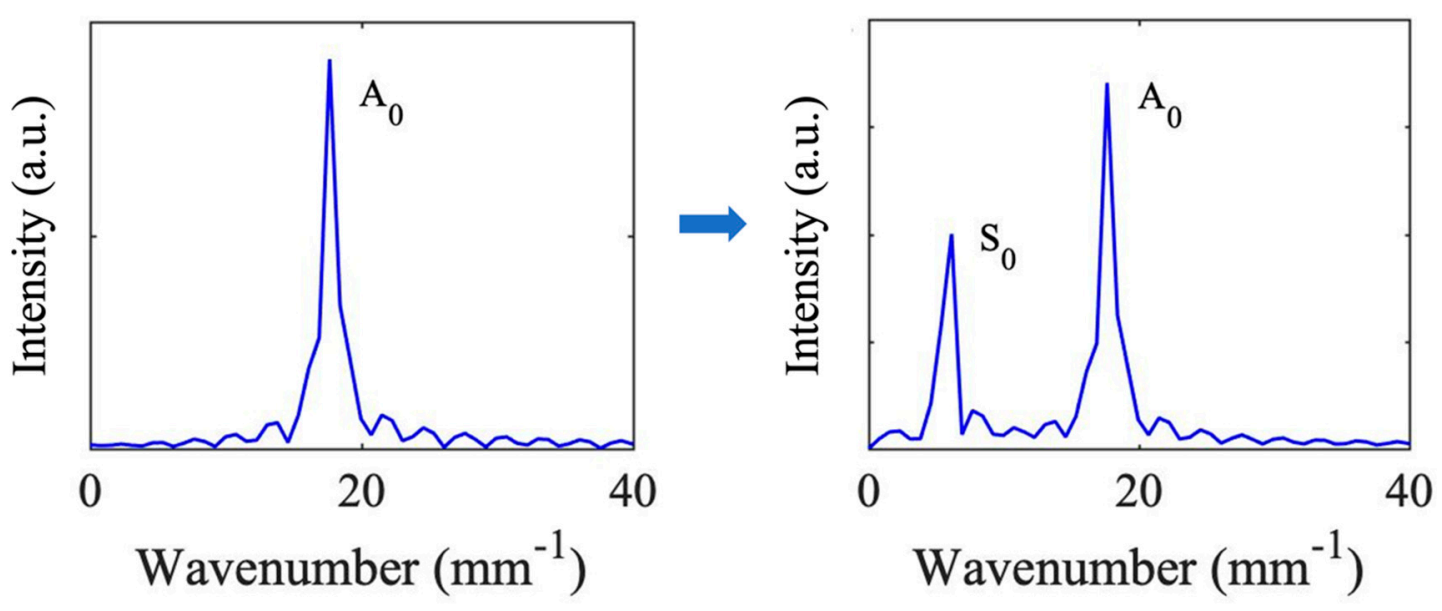

Figure 4. Illustration of the Fourier transformation of mode conversion of an $\mathrm{A}_{0}$ incident Lamb mode into both $\mathrm{A}_{0}$ and $\mathrm{S}_{0}$ modes after traversing the asymmetric-pillared meta-plate at $5 \mathrm{MHz}$. The figures show the discrete fast Fourier Transformation (FFT) of the out-of-plane displacement within an area on top of the plate. The left figure corresponds to the incident $A_{0}$ Lamb wave and the right figure refers to the transmitted wave containing both of the $\mathrm{S}_{0}$ and $\mathrm{A}_{0}$ Lamb modes.

\section{Asymmetric Propagation of Pillared Meta-Plates}

To verify the asymmetric propagation behavior, the forward (black) and backward (red) transmission with either $\mathrm{A}_{0}$ or $\mathrm{S}_{0}$ mode excitations are shown in the left and right panels of Figure 5, respectively. The $A_{0}$ and $S_{0}$ Lamb waves are excited by a force along the $z$ direction and $x$ direction, respectively, in a cross-section of the plate. Since the dominant displacement components for $\mathrm{A}_{0}$ and $\mathrm{S}_{0}$ modes are $u_{z}$ and $u_{x}$, respectively, it is not correct to directly adopt the same transmission definition as in Figures 2 and 3 due to the occurrence of a mode conversion in the combined structures. Instead, we employ the transmission definition in Figure 5 as $20 \log \left(|u| /\left|u_{\text {ref }}\right|\right)$, where $|u|$ is the volume integrated absolute value of the total displacement of the pillared plate in the downstream while $\left|u_{\text {ref }}\right|$ is the same implementation in the reference plate without any pillar. Let us first consider an $\mathrm{A}_{0}$ incident field (Figure 5a). In the backward direction, the symmetric-pillared structure prohibits the propagation in Zone 2 and the transmission drops to zero. However, in the forward direction, the asymmetric-pillared structure breaks the symmetry of the incident wave and allows the final transmission of the symmetric part of the waves. Let us notice a huge difference of $200 \mathrm{~dB}$ between the forward and backward directions. A similar analysis can be applied to an $\mathrm{S}_{0}$ excitation where the transmission is asymmetric in Zones 1 and 3. To discuss the phenomenon of mode conversion during the forward transmissions with $S_{0}$ and $A_{0}$ excitations, two complementary illustrations are given in Figure 6 . In the first case of $\mathrm{S}_{0}$ excitation (Figure $6 \mathrm{a}$ ), an FFT analysis of the displacement field shows that the wave vector of the transmitted wave corresponds to an $A_{0}$ wave. In the case of $A_{0}$ excitation, we present in Figure $6 \mathrm{~b}$ the representation of the wave in real space showing the conversion of the incident wave to an $S_{0}$ transmitted wave.

For the $S_{0}$ mode incidence in Figure $5 b$, the asymmetric propagation of Frequency Zone 1 (2.9-3.5 MHz) has a big relative bandwidth of $f / f_{c}=0.19$, where $f$ and $f_{c}$ are the zone width and center zone frequency, respectively. The average value of the forward transmission in Zone 1 is $-10.8 \mathrm{~dB}$ which corresponds to a sufficiently high efficiency. Additionally, the wavelength of the $S_{0}$ mode Lamb wave at $3 \mathrm{MHz}$ is about $2000 \mu \mathrm{m}, 20$ times bigger than the lattice constant $a=100 \mu \mathrm{m}$, showing that the size of the pillar unit cell is a deep subwavelength. Therefore, we can conclude that our proposal provides an efficient broadband subwavelength device. Additionally, the whole size of the device remains in this case smaller than the wavelength. 


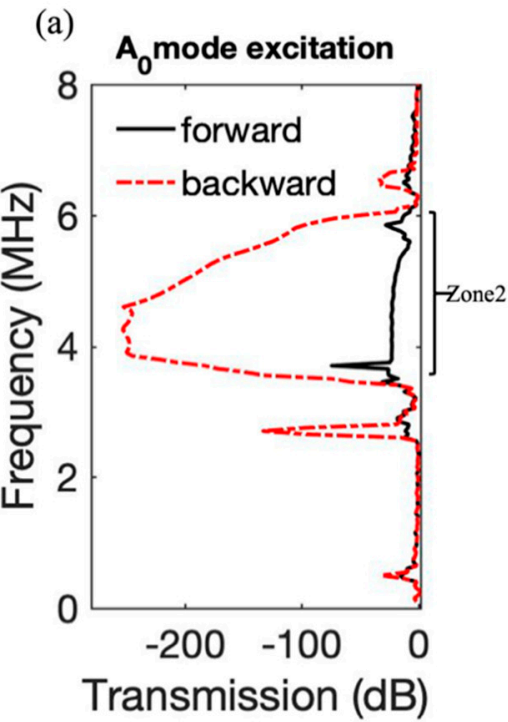

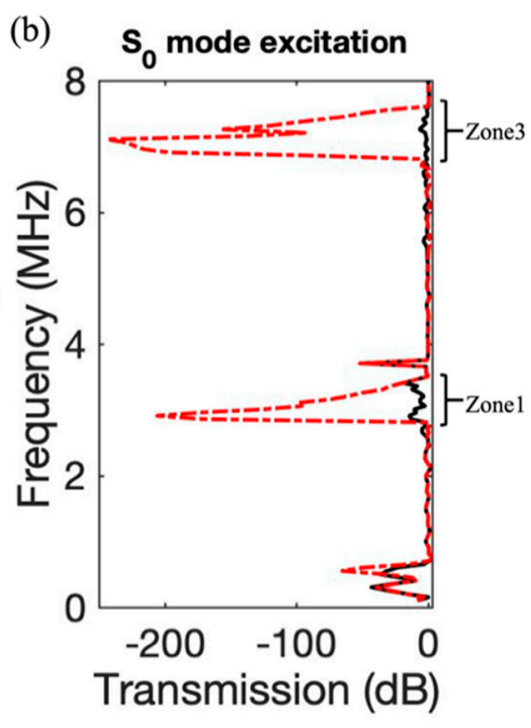

Figure 5. Transmission of the $A_{0}(\mathbf{a})$ and $S_{0}(\mathbf{b})$ Lamb mode excitations in forward and backward directions. The backward transmission is prohibited in Zone 2 for $\mathrm{A}_{0}$ excitation and in Zones 1 and 3 for $S_{0}$ excitation, while the forward transmission is allowed in these frequency ranges.

(a)
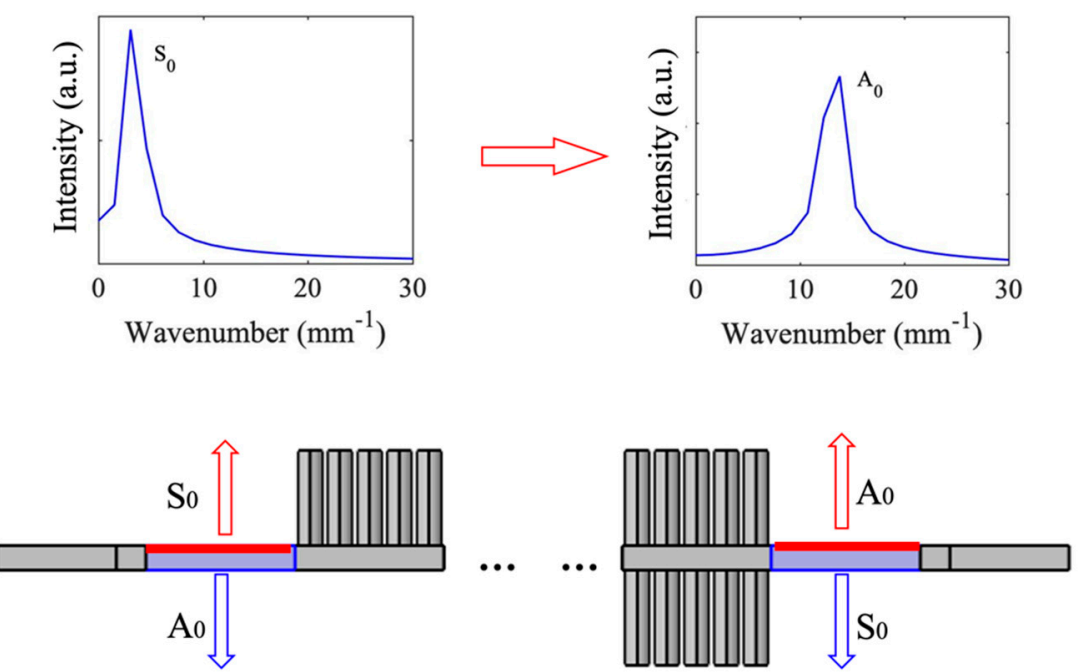

(b)

Figure 6. Conversion of the incident wave in forward transmission: (a) the excited $\mathrm{S}_{0}$ Lamb mode is converted to the $\mathrm{A}_{0}$ Lamb mode at $3 \mathrm{MHz}$ by FFT; $(\mathbf{b})$ the excited $\mathrm{A}_{0}$ Lamb mode is converted to the $\mathrm{S}_{0}$ Lamb mode at $5 \mathrm{MHz}$ by the plate's vibrations (side view of the plate).

For the $\mathrm{A}_{0}$ mode incidence in Figure $5 \mathrm{a}$, the asymmetric propagation of Frequency Zone 2 (3.8-6 MHz) has a bigger relative bandwidth as $f / f_{c}=0.45$ and the wavelength of the $A_{0}$ mode Lamb wave at $4 \mathrm{MHz}$ is about $450 \mu \mathrm{m}$, i.e., 4.5 times bigger than the lattice constant $a$. The average forward transmission is $-19 \mathrm{~dB}$ which is relatively low and needs to be improved. An alternative solution will be proposed in Section 5 .

\section{Alternative Designs for Asymmetrical Transmission}

The design proposed an asymmetric propagation based on the combination of two structures: a symmetrical-pillared structure displaying band gaps independently for each symmetry of the modes 
and an asymmetrical pillared structure, which plays the role of a mode converter due to the mixing of the Lamb modes. Keeping the symmetrical structure unchanged, the asymmetrical part can be realized in several other ways for which the performance needs to be evaluated. Below, we consider two other examples, assuming the incident wave is an A0 Lamb mode (Figure 7). With respect to the example of Section 4, called Case 1, in Cases 2 and 3 the asymmetric part constitutes a two-sided pillared array where the lower pillar has a different height $h_{\text {lower }}=0.8 h=256 \mu \mathrm{m}$ or a different diameter $d_{\text {lower }}$ $=0.7 d=56 \mu \mathrm{m}$ from the upper pillar, respectively. Similar asymmetric propagations are achieved, as demonstrated in Figure 7. For forward transmission in Frequency Zone 2 (3.8-6 MHz), on one hand, the transmission curve of Case 3 is almost the same as that of Case 1; on the other hand, it appears that there are some oscillations near $4 \mathrm{MHz}$ for Case 2, while the average value is similar as the other two cases. Therefore, diverse structures can be proposed to design the asymmetric part.

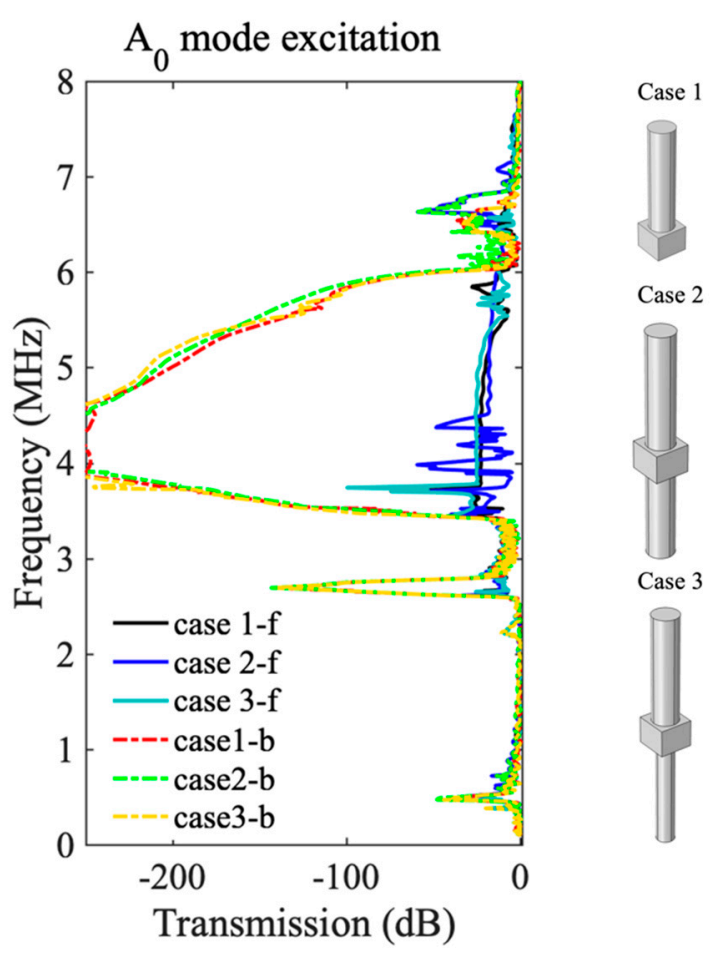

Figure 7. For $A_{0}$ Lamb mode excitation, the forward (-f) and backward (-b) transmission curves of 3 cases of asymmetric parts, as shown on the right, where Case 1 is made up of single-sided pillars as in Figure 5 a with $h=320 \mu \mathrm{m}, d=80 \mu \mathrm{m}$, Case 2 is a different pillar attached to the lower part as $h_{\text {lower }}$ $=0.8 \mathrm{~h}=256 \mu \mathrm{m}, d_{\text {lower }}=d=80 \mu \mathrm{m}$, and Case 3 is a different pillar attached to the lower part as $h_{\text {lower }}$ $=h=320 \mu \mathrm{m}, d_{\text {lower }}=0.7 d=56 \mu \mathrm{m}$.

To strengthen the mode conversion, we further propose the introduction of disorder in the asymmetric part, as in Case 4, when the height of the pillar is randomly chosen with the standard uniform distribution, even including blind holes with random finite depths, as shown in the inset of Figure 8. Since the low backward transmission will be always guaranteed with the double-sided pillar filter, we plotted only the comparison of the forward transmission between Cases 1 and 4 in Figure 8 . Obviously, the transmission of Case 4 in Frequency Zone 2 is improved, and the average value is $-10.4 \mathrm{~dB}$, compared to $-19 \mathrm{~dB}$ for Case 1 , supporting that disorder in asymmetric structure promotes the forward transmission level. Finally, it is worth noting that the studied mechanism of asymmetric propagation for the Lamb waves can also be applied to other directions in two-dimensional plates, as supported by the dispersion curve analysis in Figures 2 and 3 . 


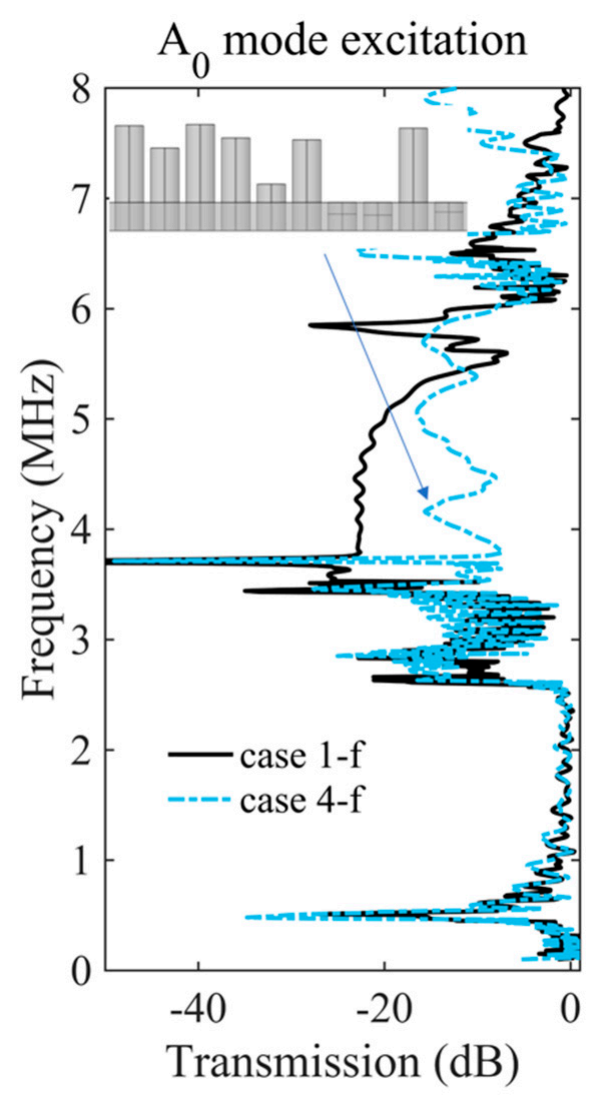

Figure 8. $A_{0}$ Lamb mode excitation. The forward (-f) transmission curves of Cases 1 and 4 of the asymmetric part, where Case 4 is a disorder profile of pillars and blind holes in the plate as shown in the inset.

Finally, special attention should be paid to the utilization of the terms "asymmetric" and "nonreciprocal" wave propagations. They are not equivalent, which means that nonreciprocity can induce asymmetric wave propagation but the inverse is not necessarily true [26]. In a reciprocal system, asymmetric wave propagation can happen with a linear mode-conversion mechanism, which is the case in this work. Meanwhile, the discussion between the terms "diode" and "reciprocity" can be found in [44], where it is stressed that a linear structure which obeys reflection-transmission reciprocity should not strictly be called a diode. Considering that other terms such as "unidirectional" or "one-way propagation" have also been used in the literature, we finally believe the term "asymmetric propagation" would be the most proper way to express the transmission behaviors presented in this study.

\section{Summary}

In summary, we proposed a meta-plate consisting of a combination of symmetrically and asymmetrically sided pillars to realize the asymmetric propagation of Lamb waves with targeted objects as subwavelengths, broadband, high efficiency and simple structures. The symmetrically sided pillars are able to decouple the $\mathrm{A}_{0}$ and $\mathrm{S}_{0}$ Lamb modes while the asymmetrical pillars hybridize them. These hybridized modes by asymmetric structures convert either $\mathrm{A}_{0}$ or $\mathrm{S}_{0}$ Lamb modes into their coexistence. The symmetric structure provides a selective low frequency and broad band gaps for $\mathrm{A}_{0}$ or $\mathrm{S}_{0}$ Lamb modes. Therefore, asymmetric propagation is realized by combining the two structures in the meta-plate and the difference of asymmetric propagation is over $200 \mathrm{~dB}$. Moreover, an FFT analysis of wavenumbers and plate vibrations are carried out to clearly support the mechanism of the mode conversion in the proposed meta-plate. We further introduce disorder in the asymmetric structure part to efficiently enhance the forward transmission level for the $\mathrm{A}_{0}$ Lamb mode incidence. In addition, the entire length of the meta-plate can possibly be kept below the operating wavelength, 
making it as an asymmetric acoustic metasurface. By choosing the proper geometric parameters, asymmetric propagation can be realized at other desired frequency ranges. The proposed pillared meta-plates are good candidates for wave isolation, non-destructive evaluations and signal processing, among others.

Author Contributions: Conceptualization, W.W. and Y.J.; Investigation, L.C.; Writing-Original Draft Preparation, W.W., Y.J., D.T., and B.D.-R.; Writing_-Review and Editing, L.C., W.W., G.N., Y.J., D.T., B.D.-R. All authors have read and agreed to the published version of the manuscript.

Funding: This research received no external funding.

Acknowledgments: This work was supported by the National Natural Science Foundation of China (Grant No. 11902223), the Shanghai Pujiang Program (Grant No. 19PJ1410100), the program for professor of special appointment (Eastern Scholar) at Shanghai Institutions of Higher Learning and the Fundamental Research Funds for the Central Universities. Daniel Torrent acknowledges financial support through the "Ramón y Cajal" fellowship under grant number RYC-2016-21188 and to the Ministry of Science, Innovation and Universities through Project No. RTI2018- 093921-A-C42.

Conflicts of Interest: The authors declare no conflict of interest.

\section{References}

1. Pennec, Y.; Djafari-Rouhani, B.; Larabi, H.; Vasseur, J.; Hladky-Hennion, A. Low-frequency gaps in a phononic crystal constituted of cylindrical dots deposited on a thin homogeneous plate. Phys. Rev. B 2008, 78, 104105. [CrossRef]

2. Wu, T.-T.; Huang, Z.-G.; Tsai, T.-C.; Wu, T.-C. Evidence of complete band gap and resonances in a plate with periodic stubbed surface. Appl. Phys. Let. 2008, 93, 111902. [CrossRef]

3. Pennec, Y.; Vasseur, J.O.; Djafari-Rouhani, B.; Dobrzyński, L.; Deymier, P.A. Two-dimensional phononic crystals: Examples and applications. Surf. Sci. Rep. 2010, 65, 229-291. [CrossRef]

4. Hussein, M.I.; Leamy, M.J.; Ruzzene, M. Dynamics of phononic materials and structures: Historical origins, recent progress, and future outlook. Appl. Mech. Rev. 2014, 66, 040802. [CrossRef]

5. Ma, G.; Sheng, P. Acoustic metamaterials: From local resonances to broad horizons. Sci. Adv. 2016, 2, e1501595. [CrossRef] [PubMed]

6. Cummer, S.A.; Christensen, J.; Alù, A. Controlling sound with acoustic metamaterials. Nat. Rev. Mater. 2016, 1, 16001. [CrossRef]

7. Jin, Y.; Djafari-Rouhani, B.; Torrent, D. Gradient index phononic crystals and metamaterials. Nanophotonics 2019, 8, 685-701. [CrossRef]

8. Assouar, B.; Liang, B.; Wu, Y.; Li, Y.; Cheng, J.-C.; Jing, Y. Acoustic metasurfaces. Nat. Rev. Mater. 2018, 3, 460-472. [CrossRef]

9. Huang, S.; Zhou, Z.; Li, D.; Liu, T.; Wang, X.; Zhu, J.; Li, Y. Compact broadband acoustic sink with coherently coupled weak resonances. Sci. Bull. 2019, 65, 373-379. [CrossRef]

10. Jin, Y.; Kumar, R.; Poncelet, O.; Mondain-Monval, O.; Brunet, T. Flat acoustics with soft gradient-index metasurfaces. Nat. Commun. 2019, 10,1-6. [CrossRef]

11. Jin, Y.; Bonello, B.; Moiseyenko, R.P.; Pennec, Y.; Boyko, O.; Djafari-Rouhani, B. Pillar-type acoustic metasurface. Phys. Rev. B 2017, 96, 104311. [CrossRef]

12. Kafesaki, M.; Sigalas, M.M.; García, N. Frequency Modulation in the Transmittivity of Wave Guides in Elastic-Wave Band-Gap Materials. Phys. Rev. Lett. 2000, 85, 4044-4047. [CrossRef] [PubMed]

13. Jin, Y.; Fernez, N.; Pennec, Y.; Bonello, B.; Moiseyenko, R.P.; Hémon, S.; Pan, Y.; Djafari-Rouhani, B. Tunable waveguide and cavity in a phononic crystal plate by controlling whispering-gallery modes in hollow pillars. Phys. Rev. B 2016, 93, 054109. [CrossRef]

14. Celli, P.; Gonella, S. Manipulating waves with LEGOßbricks: A versatile experimental platform for metamaterial architectures. Appl. Phys.Lett. 2015, 107, 081901. [CrossRef]

15. Jin, Y.; Pennec, Y.; Pan, Y.; Djafari-Rouhani, B. Phononic crystal plate with hollow pillars actively controlled by fluid filling. Crystals 2016, 6, 64. [CrossRef]

16. Ke, M.; Zubtsov, M.; Lucklum, R. Sub-wavelength phononic crystal liquid sensor. J. Appl. Phys. 2011, 110, 026101. [CrossRef] 
17. Pennec, Y.; Jin, Y.; Djafari-Rouhani, B. Phononic and photonic crystals for sensing applications. Adv. Appl. Mech. Elsevier 2019, 52, 105-145.

18. Jin, Y.; Torrent, D.; Pennec, Y.; Pan, Y.; Djafari-Rouhani, B. Simultaneous control of the S0 and A0 Lamb modes by graded phononic crystal plates. J.Appl. Phys. 2015, 117, 244904. [CrossRef]

19. Wang, W.; Bonello, B.; Djafari-Rouhani, B.; Pennec, Y. Topological valley, pseudospin, and pseudospin-valley protected edge states in symmetric pillared phononic crystals. Phys. Rev. B 2019, 100, 140101. [CrossRef]

20. Jin, Y.; Torrent, D.; Djafari-Rouhani, B. Robustness of conventional and topologically protected edge states in phononic crystal plates. Phys. Rev. B 2018, 98, 054307. [CrossRef]

21. Wang, W.; Jin, Y.; Wang, W.; Bonello, B.; Djafari-Rouhani, B.; Fleury, R. Robust Fano resonance in a topological mechanical beam. Phys. Rev. B 2020, 101, 024101. [CrossRef]

22. Wang, Z.; Yu, S.-Y.; Liu, F.-K.; Tian, Y.; Gupta, S.K.; Lu, M.-H.; Chen, Y.-F. Slow and robust plate acoustic waveguiding with valley-dependent pseudospins. Appl. Phys. Express 2018, 11, 107301. [CrossRef]

23. Zhang, X.; Xiao, M.; Cheng, Y.; Lu, M.-H.; Christensen, J. Topological sound. Commun. Phys. $2018,1,97$. [CrossRef]

24. Ma, G.; Xiao, M.; Chan, C. Topological phases in acoustic and mechanical systems. Nat. Rev. Phys. 2019, 1, 281-294. [CrossRef]

25. Jin, Y.; Wang, W.; Wen, Z.; Torrent, D.; Djafari-Rouhani, B. Topological states in twisted pillared phononic plates. Extrem. Mech. Lett. 2020, 39, 100777. [CrossRef]

26. Nassar, H.; Yousefzadeh, B.; Fleury, R.; Ruzzene, M.; Alù, A.; Daraio, C.; Norris, A.N.; Huang, G.; Haberman, M.R. Nonreciprocity in acoustic and elastic materials. Nat. Rev. Mater. 2020. [CrossRef]

27. Fleury, R.; Sounas, D.L.; Sieck, C.F.; Haberman, M.R.; Alù, A. Sound isolation and giant linear nonreciprocity in a compact acoustic circulator. Science 2014, 343, 516-519. [CrossRef]

28. Zhu, X.; Li, J.; Shen, C.; Peng, X.; Song, A.; Li, L.; Cummer, S.A. Non-reciprocal acoustic transmission via space-time modulated membranes. Appl. Phys. Lett. 2020, 116, 034101. [CrossRef]

29. Trainiti, G.; Ruzzene, M. Non-reciprocal elastic wave propagation in spatiotemporal periodic structures. New J. Phys. 2016, 18, 083047. [CrossRef]

30. Trainiti, G.; Xia, Y.; Marconi, J.; Cazzulani, G.; Erturk, A.; Ruzzene, M. Time-periodic stiffness modulation in elastic metamaterials for selective wave filtering: Theory and experiment. Phys. Rev. Lett. 2019, 122, 124301. [CrossRef]

31. Huang, J.; Zhou, X. A time-varying mass metamaterial for non-reciprocal wave propagation. Int. J. Solids Struct. 2019, 164, 25-36. [CrossRef]

32. Wang, Y.; Yousefzadeh, B.; Chen, H.; Nassar, H.; Huang, G.; Daraio, C. Observation of nonreciprocal wave propagation in a dynamic phononic lattice. Phys. Rev. Lett. 2018, 121, 194301. [CrossRef] [PubMed]

33. Chen, Y.; Li, X.; Nassar, H.; Norris, A.N.; Daraio, C.; Huang, G. Nonreciprocal Wave Propagation in a Continuum-Based Metamaterial with Space-Time Modulated Resonators. Phys. Rev. Appl. 2019, 11, 064052. [CrossRef]

34. Popa, B.-I.; Cummer, S.A. Non-reciprocal and highly nonlinear active acoustic metamaterials. Nat. Commun. 2014, 5, 3398. [CrossRef]

35. Liang, B.; Guo, X.; Tu, J.; Zhang, D.; Cheng, J. An acoustic rectifier. Nat. Mater. 2010, 9, 989-992. [CrossRef]

36. Li, F.; Anzel, P.; Yang, J.; Kevrekidis, P.G.; Daraio, C. Granular acoustic switches and logic elements. Nat. Commun. 2014, 5, 5311. [CrossRef]

37. Boechler, N.; Theocharis, G.; Daraio, C. Bifurcation-based acoustic switching and rectification. Nat. Mater. 2011, 10, 665-668. [CrossRef]

38. Li, Y.; Shen, C.; Xie, Y.; Li, J.; Wang, W.; Cummer, S.A.; Jing, Y. Tunable Asymmetric Transmission via Lossy Acoustic Metasurfaces. Phys. Rev. Lett. 2017, 119, 035501. [CrossRef]

39. Zhu, X.; Zou, X.; Liang, B.; Cheng, J. One-way mode transmission in one-dimensional phononic crystal plates. J. Appl. Phys. 2010, 108, 124909. [CrossRef]

40. Liu, T.; Zhu, X.; Chen, F.; Liang, S.; Zhu, J. Unidirectional Wave Vector Manipulation in Two-Dimensional Space with an All Passive Acoustic Parity-Time-Symmetric Metamaterials Crystal. Phys. Rev. Lett. 2018, 120, 124502. [CrossRef]

41. Zhu, J.; Zhu, X.; Yin, X.; Wang, Y.; Zhang, X. Unidirectional Extraordinary Sound Transmission with Mode-Selective Resonant Materials. Phys. Rev. Appl. 2020, 13, 041001. [CrossRef] 
42. Cao, L.; Xu, Y.; Assouar, B.; Yang, Z. Asymmetric flexural wave transmission based on dual-layer elastic gradient metasurfaces. Appl. Phys. Lett. 2018, 113, 183506. [CrossRef]

43. Jiang, X.; Liang, B.; Zou, X.-Y.; Yang, J.; Yin, L.-L.; Yang, J.; Cheng, J.-C. Acoustic one-way metasurfaces: Asymmetric phase modulation of sound by subwavelength layer. Sci. Rep. 2016, 6, 28023. [CrossRef] [PubMed]

44. Maznev, A.A.; Every, A.G.; Wright, O.B. Reciprocity in reflection and transmission: What is a 'phonon diode'? Wave Motion 2013, 50, 776-784. [CrossRef]

(C) 2020 by the authors. Licensee MDPI, Basel, Switzerland. This article is an open access article distributed under the terms and conditions of the Creative Commons Attribution (CC BY) license (http://creativecommons.org/licenses/by/4.0/). 